\title{
Active surveillance of low-risk papillary thyroid microcarcinomas
}

\author{
Yasuhiro Ito, Akira Miyauchi \\ Department of Surgery, Kuma Hospital, Kobe, Japan \\ Contributions: (I) Conception and design: All authors; (II) Administrative support: A Miyauchi; (III) Provision of study materials or patients: Y Ito; (IV) \\ Collection and assembly of data: All authors; (V) Data analysis and interpretation: Y Ito; (VI) Manuscript writing: All authors; (VII) Final approval of \\ manuscript: All authors. \\ Correspondence to: Dr. Yasuhiro Ito, MD, PhD. Department of Surgery, Kuma Hospital, 8-2-35, Shimoyamate-dori, Chuo-ku, Kobe, Hyogo 650- \\ 0011, Japan. Email: ito01@kuma-h.or.jp.
}

\begin{abstract}
In 1993, active surveillance of low-risk papillary thyroid microcarcinomas (PTMCs) started in Kuma Hospital, Kobe, Japan. It has spread globally after the publishing of American Thyroid Association (ATA) guidelines. During our hospital's active surveillance program, few patients (8.0\%) showed tumor size enlargement $\geq 3 \mathrm{~mm}$ or the new appearance of node metastasis $(3.8 \%)$ at 10 -year follow-up. Conversion surgery was recommended for patients with disease progression. To date, no patients showed significant recurrence or metastasis or died with thyroid carcinoma when patients underwent active surveillance or after surgery due to PTMC progression. Unlike clinical papillary thyroid carcinoma (PTC), elderly patients' PTMCs were less progressive compared to those of young and middle-aged patients, indicating that elderly patients are strong candidates for the active surveillance of their PTMC. Although young patients' PTMCs are the most progressive, $>50 \%$ and $>75 \%$ of patients in their 20 s and 30 s would avoid conversion surgical treatment in their lifetime, respectively (according to estimated lifetime probability), indicating that such young patients are still candidates for active surveillance. It can thus be concluded that active surveillance is appropriate to be first management for PTMCs, based on the accumulation of favorable outcomes of PTMC patients who have undergone active surveillance, as well as the lower incidences of unfavorable events and lower medical cost than immediate surgery.
\end{abstract}

Keywords: Active surveillance; papillary microcarcinoma; thyroid; patient age

Submitted Mar 03, 2020. Accepted for publication May 27, 2020.

doi: $10.21037 /$ gs-2019-catp-03

View this article at: http://dx.doi.org/10.21037/gs-2019-catp-03

\section{Introduction}

Papillary thyroid microcarcinoma (PTMC) indicates papillary thyroid carcinoma (PTC) measuring $10 \mathrm{~mm}$ or smaller regardless of whether clinical node metastasis $(\mathrm{N})$, extrathyroid extension (Ex), and distant metastasis (M) are present. Patients with PTMCs having these high-risk features should immediately undergo extensive treatment including a total thyroidectomy, lymph node dissection, radioactive iodine (RAI) therapy, and thyroid-stimulating hormone (TSH) suppression. Recently, how to manage patients with PTMCs with no high-risk features, i.e., lowrisk PTMCs (referred to hereafter as 'PTMC' in this chapter) has recently become an important issue, since this category of cases accounts for the vast majority of PTMCs in clinical practice.

Formerly, most PTMCs were found at autopsy, which is called latent carcinomas. Its incidence is high ranging $0.5-5.2 \%$ for tumors ranging from 3 to $10 \mathrm{~mm}$ in diameter (size detectable by ultrasound examination) (1). Recent improvements in the resolving power of ultrasound and the establishment of an ultrasound-guided fine-needle aspiration cytology (FNAC) technique enabled the easy detection and diagnosis of PTMC by thyroid screenings and imaging studies for other diseases [e.g., ultrasound for patients with arterial sclerosis and chest computed tomography (CT) scans to evaluate lung disease or metastasis of other carcinomas]. 
Takebe et al. described about the clinical incidence of PTMC in 1994 (2). On ultrasound screening for the thyroid on women aged $>30$ years, who visited the clinic for breast diseases, they detected thyroid carcinoma in $3.5 \%$ of the women, which were cytologically diagnosed. This incidence was not discrepant to that in autopsy studies and also 1,000 times higher than the prevalence of thyroid carcinoma in Japanese women at that time, which was 3.1 per 100,000 population. These strongly suggested that PTMCs generally lack symptoms with no or very slow growth activity, indicating that many people have PTMCs although they are unaware. The Takebe group's results also raised significant doubt whether the detection, diagnosis, and treatment of these PTMCs is truly favorable for these patients.

Taken all these together, a policy of active surveillance for PTMCs was initiated at our institute (Kuma Hospital in Kobe, Japan) in 1993 after approval by the medical staff committee (equivalent to ethics committee). Thereafter, Miyauchi et al. showed various insights into the active surveillance (3). In this review, the history of active surveillance of PTMC and the outcomes of that surveillance are described.

\section{The initiation of a policy of active surveillance of PTMCs in Japan}

Based on the above-described evidence, Dr. Akira Miyauchi at Kuma Hospital started the active surveillance of lowrisk PTMCs in 1993, which was a prospective trial. He developed the following hypotheses: (I) most PTMCs do not or very slowly grow; (II) only active surveillance can evaluate whether they have a growth activity; (III) surgery would not be too late when progression signs, e.g., tumor enlargement or novel lymph node metastasis are found during active surveillance; and (IV) immediate surgery for all PTMCs could be more harm than good (4). The Cancer Institution Hospital (Tokyo) successively started an active surveillance in 1995 under the same hypotheses.

To date, favorable outcomes of PTMC patients who underwent active surveillance have been reported. Active surveillance was accepted as a management for PTMC in the guidelines issued by the Japan Association of Endocrine Surgeons and the Japanese Society of Thyroid Surgery [currently the Japan Association of Endocrine Surgery (JAES)] (5), and it became more strongly recommended in the revised JAES guidelines published in 2018 (6). In addition, the American Thyroid Association (ATA) guidelines state that the active surveillance for PTMCs was adopted as a management strategy (7).

\section{The change in incidence and mortality rate of thyroid carcinoma}

The change in incidence and mortality rate of thyroid carcinoma is an interesting issue. The incidence of thyroid carcinoma in the United States increased 2.4 times between 1973 and 2002. Between 1975 and 2009, it increased 2.9 times, but mortality rate was stable throughout $(8,9)$. In South Korea, between 1993 and 2011, the incidence was reported to increase by 15 times because of the national screening program for thyroid cancer using ultrasound and FNAC, but thyroid carcinoma mortality was stable (10). Similar findings were reported also in various countries such as Italy, the United Kingdom, Australia, and the Nordic countries (11). These data indicate that numerous harmless PTCs were detected, diagnosed and surgically treated, resulting in unnecessary surgery for a significant number of patients worldwide.

\section{Adverse events of PTMC surgery}

A study conducted in South Korea revealed that many thyroid cancer patients who underwent surgery for this cancer suffered from adverse events damaging the QoL of patients such as vocal cord paralysis and hypoparathyroidism (10). Indeed, Oda $e t a l$. performed the comparison between the incidences of adverse events in PTMC patients who received immediate surgery (surgery group) and those in patients who received active surveillance with conversion surgery for progressive disease (active surveillance group) (12). The incidences of transient vocal cord paralysis and transient hypoparathyroidism in the surgery group were $4.1 \%$, and $16.7 \%$, which were significantly higher than these incidences in the active surveillance group at $0.6 \%$ and $2.8 \%$, respectively $(\mathrm{P}<0.0001)$. Permanent hypoparathyroidism occurred in $1.6 \%$ of the surgery group patients, which was also significantly higher than the rate in the active surveillance group $(0.08 \%, \mathrm{P}<0.0001)$. In addition, the recurrent laryngeal nerves of two patients $(0.2 \%)$ in the surgery group were injured during the surgery, resulting in permanent vocal cord paralysis.

Our hospital is a specialized hospital for the thyroid, and the surgeons at Kuma Hospital are all thyroid surgery specialists. If patients with PTMC undergo surgery by nonspecialist, the incidences of transient vocal cord paralysis 
Table 1 Indications of immediate surgery for PTMCs

\begin{tabular}{ll}
\hline Type & Reasons for surgery \\
\hline Clinically high-risk features & $\begin{array}{l}\text { 1. } \mathrm{N} \text { - and/or M-positive (very rare) } \\
\text { 2. Presence of signs or symptoms of carcinoma extension to the recurrent laryngeal nerve or the trachea } \\
\text { 3. High-grade malignancy on cytology (very rare) }\end{array}$
\end{tabular}

A feature unsuitable for active Tumors having the possibility of invading the trachea or recurrent laryngeal nerve surveillance

No evidence $\quad$ Patients $<20$ years old

PTMC, papillary thyroid microcarcinoma.

and transient hypoparathyroidism would be much higher, and this is another factor supporting the concept that active surveillance is more appropriate for PTMC patients than immediate surgery. Several insights into the active surveillance program at Kuma Hospital were introduced and summarized by Miyauchi et al. in 2018 (3).

\section{Contraindications for the active surveillance for PTMCs}

Not all PTMCs are suitable for active surveillance. The initial case selection is very important. Table 1 lists indications of immediate surgery for PTMCs. PTMCs positive for clinical lymph node metastasis and/or (although rare) distant metastasis are strong candidates for surgery. PTMCs showing vocal cord paralysis due to carcinoma invasion and those having signs of tracheal invasion on CT scan, MRI or fiberscope, namely symptomatic PTMCs, should be immediately treated. PTMCs suspected of highgrade malignancy on cytology should be surgically treated.

Active surveillance is not recommended for PTMCs having the possibility of invading the trachea and/or the recurrent laryngeal nerve, although these features do not necessarily indicate biological aggressiveness (Table 1). This is because, if a tumor grows and invades the trachea and/ or the recurrent laryngeal nerve, the patient's quality of life could be significantly damaged despite surgical treatment after invasion. For the evaluation of tracheal invasion, it is useful to determine the angles formed by the tumor surface and tracheal cartilage (13). Among the subset of PTMCs $\geq 7 \mathrm{~mm}$ with obtuse angles between the tumors, $24 \%$ significantly invaded to the trachea and laminate resection or window resection was required for curative surgery. For the evaluation of invasion to the recurrent laryngeal nerve, it is important to evaluate whether or not a normal rim between the thyroid capsule and tumor surface in the direction of the nerve is present (10). If a normal rim could be observed, immediate surgery is recommended, because $9 \%$ of PTMCs $\geq 7 \mathrm{~mm}$ without a normal rim showed a significant invasion to the recurrent laryngeal nerve and partial layer resection or resection of the nerve with anastomosis were required (13). Even for tumors located in the dorsal side of the thyroid, if a normal rim is present, the tumors are candidates for active surveillance.

In contrast, PTMCs with possible invasion of the anterior or the lateral capsule of the thyroid can undergo active surveillance. When a patient with this type of PTMC undergoes conversion surgery, a resection of the anterior muscles or adipose tissue in the lateral side may be required, but this resection does not affect the patient's quality of life. Patients having a family history of differentiated thyroid carcinoma and multiple PTMCs are not candidates for immediate surgery. These could have moderate prognostic values, but a total thyroidectomy would generally be performed for such cases. This would most likely result in an increase in the rate of surgery-related adverse events such as permanent recurrent laryngeal nerve paralysis and hypoparathyroidism. However, neither of a family history nor multiplicity of PTMCs were recognized as significant prognostic factors in our hospital's active surveillance program, as described next.

\section{Active surveillance program at Kuma Hospital}

Now, we introduce actual active surveillance practice for low-risk PTMCs in our hospital. First, for suspicious nodules $\geq 5 \mathrm{~mm}$, we perform an FNAC. This is in sharp contrast to the 2015 ATA guidelines that do not recommend an FNAC for nodules $\leq 10 \mathrm{~mm}$ having no malignant features such as symptoms and lymph node/distant 


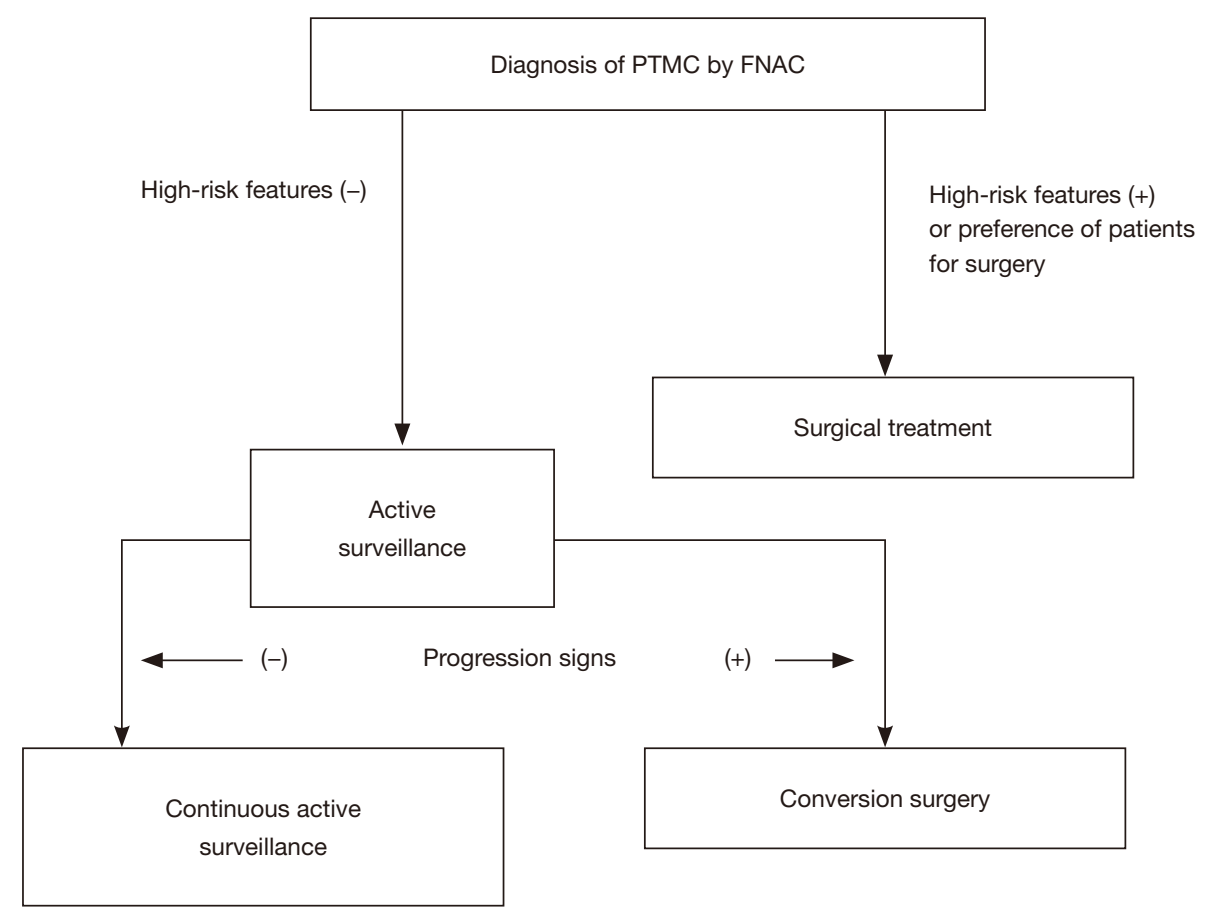

Figure 1 Algorithm for active surveillance of PTMC. PTMC, papillary thyroid microcarcinoma.

metastasis (7). The authors of the ATA guidelines may have assumed that patients in the U.S. could not live with cancer by active surveillance. In contrast, most Japanese institutions, including ours perform FNAC of nodules (generally $\geq 5 \mathrm{~mm}$ ), which are suspected of being PTMC. If the nodules are cytologically diagnosed as PTMCs, we inform the diagnosis to the patients. If we should not diagnose them on cytology, they might possibly come to other clinics, receive FNAC, be diagnosed as having PTMC, and receive unnecessary surgery. We might also be subjected to bad publicity for overlooking thyroid carcinomas, and this would be unfavorable for both patients and our hospital. We set the tumor cutoff size for cytological examination at $5 \mathrm{~mm}$ based on the guidebook published by the Fapan Association of Breast and Thyroid Sonology.

In the past, we equally present two management options, active surveillance and immediate surgery, to patients, and asked them to choose. However, a significant amount of favorable data about active surveillance has accumulated, and we now recommend active surveillance to the patients as the first-line management. The proportion of patients who preferred active surveillance has increased with time, and now most patients ( $>95 \%)$ choose active surveillance in Kuma Hospital. Importantly, whether patients undergo active surveillance or immediate surgery is a patient choice as a shared decision-making. Also, patients who chose active surveillance can change their minds freely and can undergo conversion surgery at any time.

Figure 1 illustrates the simple algorithm for active surveillance. Patients who make the choice to undergo active surveillance are asked to visit Kuma Hospital in another 6 months and at least once/year thereafter for a regular checkup with ultrasound for the determination of whether progression signs, tumor enlargement, and/or a novel appearance of lymph node metastasis are present. A tumorsize increase $\geq 3 \mathrm{~mm}$ compared with the tumor's size at the initiation of active surveillance is judged as enlargement. However, if the patient prefers, active surveillance is continued until the tumor reaches $13 \mathrm{~mm}$. This is because the maximum size suitable for active surveillance is $10 \mathrm{~mm}$, and definition of tumor growth is enlargement by $\geq 3 \mathrm{~mm}$. If a lymph node suspected of metastasis has newly appeared, we perform an FNAC and also measure the thyroglobulin level in the washout of the needles used for the FNAC. If metastasis is diagnosed, we perform conversion surgery.

\section{Studies of active surveillance in Japan}

In 2003, Kuma Hospital published the first study regarding active surveillance, showing that more than $70 \%$ of PTMCs 
did not progress (14). In 2010, second report was published demonstrating that the 5- and 10-year enlargement rates were $6.4 \%$ and $15.9 \%$ and the 5 - and 10 -year rates of appearance of lymph node metastasis were $1.5 \%$ and $3.4 \%$, respectively (15). In 2014, third report enrolling 1,235 patients and observed was published, which showed that 5and 10 -year enlargement rates were $4.9 \%$ and $8.0 \%$ and 5 - and 10-year cumulative rates of appearance of lymph node metastasis were $1.7 \%$ and $3.8 \%$, respectively (16). In the subset analysis of patient age at presentation, tumor enlargement rate significantly decreased with old age: the 5- and 10 -year rates were $9.1 \%$ and $12.1 \%, 5.0 \%$ and $9.1 \%$, and $4.0 \%$ and $4.1 \%$ for patients aged $<40$ years, $41-$ 59 years, and aged $\geq 60$ years, respectively. Also, the rates of novel appearance of lymph node metastasis were inversely related to patient age: the 5 - and 10 -year rates were $5.2 \%$ and $16.1 \%$ for patients aged $<40$ years, $1.4 \%$ and $2.3 \%$ for patients aged $40-59$ years, and $0.5 \%$ and $0.5 \%$ for patients aged $\geq 60$ years, respectively. On multivariate analysis, only young age $(<40$ years) independently predicted PTMC progression, whereas a family history and multiplicity of PTMCs were not predictive; it is therefore appropriate that enrolling patients with multiple PTMCs and/or a family history for active surveillance (16).

Sugitani et al. (17) in Cancer Institute Hospital (Tokyo, Japan) demonstrated that the tumor enlargement rate and lymph node metastasis appearance rate were only $7 \%$ and $1 \%$, respectively. They published a second report showing similar data for 360 patients in 2019 (18). They also investigated the appropriateness of active surveillance for T1bN0M0 PTC, and concluded that active surveillance can be an option for selected patients with T1bN0M0 PTC (18).

\section{The active surveillance for PTMCs in other countries}

Brito et al. (19) in the Memorial Sloan Kettering Cancer Center (New York, NY) in published a clinical framework for active surveillance in 2016 as a collaborative study with Kuma Hospital. Authors classified PTMCs into three categories for active surveillance: ideal, appropriate, and inappropriate. The inappropriate characteristics of active surveillance are as follows: (I) evidence of aggressive cytology; (II) subcapsular locations adjacent to the recurrent laryngeal nerve; (III) evidence of extrathyroidal extension; (IV) clinical evidence of invasion of the recurrent laryngeal nerve or trachea; (V) N1 disease; (VI) M1 disease; or (VII) a documented $\geq 3$-mm increase in the size of a confirmed
PTC tumor. These characteristics are similar to the contraindications for active surveillance of PTMCs used at Kuma Hospital.

In 2017 , the first prospective study for the active surveillance was conducted by Tuttle et al. (20), enrolling 291 cases of low-risk PTCMs (actually PTC measuring $15 \mathrm{~mm}$ or smaller). They evaluated tumor enlargement not only by maximal diameter but also by tumor volume. They demonstrated that, although only $3.8 \%$ of the tumors increased in size by $\geq 3 \mathrm{~mm}, 12.7 \%$ of the tumors increased in the volume of tumor by $>50 \%$. Tumor volume decreased by $>50 \%$ was seen in $6.7 \%$ and stable volume was observed in $80.2 \%$ of the tumors. Similar to the results in Kuma Hospital, young patients had stronger growth activity. Based on their findings, Tuttle et al. contended that the evaluation of the tumor volume is more useful than the evaluation of the maximal diameter for selecting PTMCs with high growth activity at an earlier phase for a closer follow-up schedule.

Several studies about this issue have been conducted in South Korea; the first was a study of passive (but not active) surveillance, in a retrospective analysis of the cases of PTMC patients who did not receive surgery and were followed for $>1$ year for various reasons (e.g., the patient's refusal of surgery; the presence of other, untreated malignancies; or being a poor candidate for general anesthesia) (21). The study revealed that, based on the evaluation by tumor volume, $14.1 \%$ of the PTMCs were judged as enlarged, and this value is much higher than the incidence of enlargement based on maximal tumor size at $2.1 \%$ (21). According to a South Korean multicenter study enrolling 370 patients (22), the incidence of enlargement was $23.2 \%$ based on a $\geq 50 \%$ tumor volume increase but $3.5 \%$ based on a $\geq 3-\mathrm{mm}$ increase in the tumor maximal diameter (a median period of follow-up, 32.5 months). Importantly, in that patient series, the incidence of patients who received conversion surgery was $15.7 \%$ due to patient anxiety $(37.9 \%)$, tumor enlargement $(32.8 \%)$, and appearance of lymph node metastasis $(8.6 \%)$.

Sanabria (23) performed active surveillance and showed that the overall stability rate without tumor growth $>3 \mathrm{~mm}$ was $98 \%$ at 12 months. Also, Smulever et al. (24) reported that the incidence of patients who chose active surveillance was only $25 \%$ and approx. $10 \%$ of them could not continue the active surveillance because of anxiety. However, their data were favorable that only $17 \%$ of PTMCs showed enlargement (median follow-up period of 4.6 years). A single-institution study from Italy showed that only $3 \%$ of 
Table 2 Summary of the results of active surveillance for PTMC in representative studies

\begin{tabular}{|c|c|c|c|c|c|c|c|}
\hline Affiliation & Study design & $\begin{array}{l}\text { Number of } \\
\text { patients }\end{array}$ & $\begin{array}{l}\text { Mean follow-up } \\
\text { period }\end{array}$ & $\begin{array}{l}\text { Rates of size } \\
\text { enlargement }\end{array}$ & $\begin{array}{c}\text { Rates of } \\
\text { appearance of } \\
\text { node metastasis }\end{array}$ & $\begin{array}{l}\text { Number of patients } \\
\text { who underwent } \\
\text { conversion surgery }\end{array}$ & $\begin{array}{c}\text { Number of patients } \\
\text { who died of thyroid } \\
\text { carcinoma }\end{array}$ \\
\hline $\begin{array}{l}\text { Kuma Hospital } \\
\text { (16) }\end{array}$ & Prospective & 1,235 & $\begin{array}{c}60 \text { [18-228] } \\
\text { months }\end{array}$ & $\begin{array}{l}8.0 \% \text { at } 10-y e a r \\
\text { follow-up }\end{array}$ & $\begin{array}{l}3.8 \% \text { at } 10-y e a r \\
\text { follow-up }\end{array}$ & $191(16 \%)$ & 0 \\
\hline $\begin{array}{l}\text { Cancer } \\
\text { Institute } \\
\text { Hospital* (18) }^{*}\end{array}$ & Prospective & 360 & $7.9[1-17]$ years & $\begin{array}{c}8 \% \text { after follow- } \\
\text { up }\end{array}$ & $1 \%$ after follow-up & NA & 0 \\
\hline $\begin{array}{l}\text { Memorial } \\
\text { Sloan Kettering } \\
\text { Cancer } \\
\text { Center }^{\star \star}(20)\end{array}$ & Prospective & 290 & $\begin{array}{l}25[6-166] \\
\text { months }\end{array}$ & $\begin{array}{l}12.1 \% \text { at } 5 \text {-year } \\
\text { follow-up }\end{array}$ & NA & $10(3 \%)$ & 0 \\
\hline
\end{tabular}

${ }^{*}$, data about T1aNOM0 patients were extracted. ${ }^{* *}$, enrolled patients with PTC $\leq 15 \mathrm{~mm}$.

patients (three of 93) progressed during active surveillance [median follow-up, 19 months (range, 6-54 months)], concluding that active surveillance is a useful and safe management (25).

Table 2 summarizes the outcomes of previous representative studies regarding active surveillance from high-volume centers. A perfect agreement and excellent reproducibility were detected in all these studies. One important point is that none of the patients showed a distant metastasis or died of thyroid carcinoma during active surveillance. Also, none of the patients showed a lifethreatening recurrence or died of thyroid carcinoma after conversion surgery.

\section{Evaluating tumor growth: maximal diameter vs. tumor volume}

The question of which of the two evaluations, maximal diameter or tumor volume, is better for active surveillance is thus fundamental. As indicated above, U.S. and Korean research groups recommended evaluations based on the tumor volume, proposing that this strategy can more rapidly identify PTMC that have high growth activity (20-22). Japanese physicians have traditionally used the maximal diameter for evaluating tumor growth, and a $\geq 3-\mathrm{mm}$ increase in the tumor's maximal diameter is considered to be tumor growth.

Suppose that a tumor measuring $6 \mathrm{~mm} \times 6 \mathrm{~mm} \times 6 \mathrm{~mm}$ grew to $7 \mathrm{~mm} \times 7 \mathrm{~mm} \times 7 \mathrm{~mm}$; the increase of tumor volume would be as high as $59 \%$. If tumor volume $\geq 50 \%$ is judged as enlargement, a patient showing this growth would be advised to undergo conversion surgery. However, this is definitely too early a time point for conversion surgery. Indeed, Oh et al. showed that the incidence of patients who underwent conversion surgery in their series was $15.7 \%$, which is high for a short follow-up period (median 32.5 months) (22). At Kuma Hospital, the incidence of conversion surgery with a much longer follow-up period (75 months) was $15.5 \%$, and the incidence of conversion surgery significantly decreased over time. More importantly, Japanese institutions such as Kuma Hospital and the Cancer Institute Hospital adopted the policy of using tumor enlargement based on a maximal diameter increase of $\geq 3 \mathrm{~mm}$, and distant metastasis/recurrence or carcinoma death during active surveillance or after conversion surgery have not been observed. This strongly suggests the appropriateness of the evaluation of the tumors' growth by measuring the maximal diameter, which is also much easier 
in practice than calculating the tumor volume.

\section{The natural history of PTMCs}

Miyauchi et al. (26) investigated the 10-year disease progression rate of PTMC in active surveillance for agedecade groups from the 20 s to the 70 s at diagnosis. Then, they used these rates and estimated the lifetime probability of PTMC progression for each age-decade group at presentation: $48.6 \%, 25.3 \%, 20.9 \%, 10.3 \%, 8.2 \%$, and $3.5 \%$ for patients in their $20 \mathrm{~s}, 30 \mathrm{~s}, 40 \mathrm{~s}, 50 \mathrm{~s}, 60 \mathrm{~s}$, and $70 \mathrm{~s}$, respectively. One might think that the probabilities of disease progression in patients with 20s and 30s are high for accepting active surveillance, but, in other words, more than half of the patients in the 20s and three-quarters of patients in the 30s could avoid surgery in their lifetime. We thus consider young PTMC patients (in their 20s and 30s) candidates for active surveillance if they prefer (26).

To evaluate the change in tumor volume, tumor volume doubling rate was proposed also by Miyauchi et al. $(27,28)$, which is the inverse of tumor volume doubling time. The doubling time is a useful tool for evaluating the change in tumor volume, but it has two problem points. The doubling times of tumors shrinking over time are given in negative values $(<0)$. When evaluating these tumors' growth over time, discontinuity occurs between positive and negative values. In addition, the evaluation of the doubling time is opposite to that of tumor growth activity. However, the doubling rate solves these limitations. The doubling rate indicates the number of doublings of a tumor occurring per unit time. Its negative value indicates how many halvings occurred in unit time. Miyauchi et al. calculated doubling rates of the tumor volume (/year) in 169 PTMCs during the tumors' active surveillance and divided them into four groups as follows: $>0.5$ (rather rapid growth), 0.1 to 0.5 (slow growth), -0.1 to 0.1 (stable), and less than -0.1 (decrease in growth). Of the 169 PTMCs, 5, 38, 97, and 29 were classified into rather rapid growth, slow growth, stable status, and a decrease in growth, respectively (29). Important issue is that the tumor volume of $17 \%$ of tumors decreased when patients underwent active surveillance. Moreover, the incidence of tumors having rather rapid growth plus those having slow growth decreased with patients' age: $40 \%$ in patients aged $<40$ years, $29 \%$ in those aged $40-59$ years, and $17 \%$ in those $\geq 60$ years (28).

Also, Miyauchi et al. (28) figured the 'hypothetical' tumor volume doubling rate of PTMCs before presentation. They assumed that a single cancer cell measuring $10 \mu \mathrm{m}$ was present at the patient's birth, which grew constantly. This value is the lowest growth rate necessary for single cell to grow to the tumor detected on ultrasound. Generally, a cancer cell would appear after patients' birth, and the real doubling rate should therefore be higher than the hypothetical doubling rate. Miyauchi et al. demonstrated that the hypothetical doubling rates before presentation were much higher than the doubling rates calculated after presentation during active surveillance (28). This suggests that PTMCs rapidly grow before presentation, and after presentation, the growth activity significantly decreases and may reach zero or less than zero (tumor shrinkage). This could be a natural course of most PTMCs.

More recently, Ito et al. investigated the change in growth activity evaluated on the tumor volume doubling rate before and after enlargement points, i.e., a size increase by $\geq 3 \mathrm{~mm}$ and a tumor volume increase by $\geq 50 \%$ (29). The doubling rates after the enlargement point of a $\geq 3$ - $\mathrm{mm}$ size increase were significantly lower than those observed before the enlargement point $(-0.091$ year vs. 0.509/year, $\mathrm{P}<0.0001)$. Similar results were obtained based on the $\geq 50 \%$ tumor volume increase $(-0.058 /$ year vs. $0.380 /$ year, $\mathrm{P}<0.0001)$. Only a small portion of the tumors continued to rather rapidly enlarge (doubling rate $>0.5 /$ year) at $7.7 \%$ and $3.8 \%$ after the enlargement points based on maximal tumor size and tumor volume, respectively. Conversely, $47.4 \%$ and $36.1 \%$ of the tumors shrank (doubling rates $<-0.1 /$ year) after the enlargement points based on tumor size and tumor volume, respectively. These findings suggest that performing conversion surgery immediately after a tumor reaches one of these enlargement points may be too hasty, and for patients who do not prefer surgery, continuous active surveillance may be better to avoid unnecessary surgery.

\section{Other clinical questions}

\section{The relationship between PTMC growth on pregnancy}

Since a copious amount of human chorionic gonadotropin is secreted when females are pregnant, whether PTMCs in young women having a chance of pregnancy is an important clinical question. Human chorionic gonadotropin has the activity similar to TSH stimulating the PTMCs' growth. Ito et al. demonstrated that four of the 51 patients (8\%) had tumor enlargement $\geq 3 \mathrm{~mm}$ during pregnancy, and two of them received conversion surgery after delivery (30). Active surveillance was continued for the remaining two patients because their PTMCs became stable after delivery. None of 
the four patients showed significant metastasis/recurrence during active surveillance or after conversion surgery. It is therefore concluded that PMTCs in young females are candidates for active surveillance.

\section{Relationship between growth activity of PTMC and ultrasound findings}

According to the report from The Cancer Institute Hospital, PTMCs having rich vascularity (regardless of the presence of calcification) or having microcalcification only had higher growth activity than those with poor vascularity and/or a high degree of calcification (31). However, they also reported that blood supply of $61.4 \%$ of the tumors having rich vascularity significantly decreased during active surveillance, and at the10-year follow-up, calcification of $51.8 \%$ of the tumors increased. A high degree of calcification and poor vascularity at the last examination were inversely related to PTMC progression in multivariate analysis, but none of these patients showed life-threatening metastasis or died of thyroid carcinoma during active surveillance or after conversion surgery. Therefore, patients with PTMCs can undergo active surveillance unless there are other risk factors, regardless of ultrasound findings.

\section{Comparison of medical costs between active-surveillance and immediate-surgery}

Oda et al. showed, subject to the Japanese Health Care Insurance System, that the 10-year cost of immediate surgery was 4.1 times that of active surveillance $(928,094$ vs. 225,695 yen/patient) (32). In this analysis, they included the cost of conversion surgery after active surveillance, and the costs of surgery for PTC recurrence and postoperative management in the immediate surgery group. Also, it was reported that active surveillance is more cost-effective than immediate surgery from Hong Kong (33). In the United States, one study indicated that immediate lobectomy for PTMC was more cost-effective than active surveillance, but their analyses were much complicated and had many assumptions (34). Taken together, the costs of active surveillance and immediate surgery vary among countries, and they should thus be calculated for each country.

\section{The effects of TSH suppression on PTMC progression}

Controversial findings were published about the relationship between TSH suppression and PTMC progression. Sugitani et al. (35) showed that TSH levels are not related to PTMC progression, but Kim et al. (36) demonstrated that a sustained elevation of TSH level results in PTMC progression. Ito et al. (15) noted that in younger patients who underwent TSH suppression, PTMCs only occasionally grew, although the PTMCs in young patients were more likely to grow than those in middle-aged and old patients. Further studies enrolling larger numbers of patients are mandatory to draw any confirmative conclusions, but a low-normal setting for the serum TSH level might be a favorable strategy for active surveillance in younger patients.

\section{Anxiety in both patients and physicians}

Anxiety for active surveillance arises not only among patients but also among physicians. To date, two Australian studies indicated that many physicians hesitate to try active surveillance because of their anxiety $(37,38)$, but such anxiety would arise mainly from a lack of experience. At Kuma Hospital, the incidence of patients who chose active surveillance was only 30\% between 1993 and 1997, but this increased over time and reached $88 \%$ in 2014 and $\geq 95 \%$ in 2018 (39). This increase occurred because the safety and effectiveness of active surveillance were firmly established over this period, and because physicians realized that unfavorable events such as vocal cord paralysis and hypoparathyroidism are not completely avoidable even by skillful surgeons (16). In addition, physicians' anxiety can significantly influence the level of their patients' anxiety.

A joint study between the Dartmouth Institute in the U.S. and Kuma Hospital showed that although $37 \%$ of patients who underwent active surveillance had worried sometimes (or even more) about their cancer, $60 \%$ of these described a significant decrease in their worrying over time. Very importantly, as much as $80 \%$ of the patients agreed or strongly agreed that their decision to undergo active surveillance matched their personal values. Moreover, $83 \%$ of the patients agreed or strongly agreed that choosing active surveillance was the best decision for themselves (40). These favorable results are because not only of the successful management but of the confident attitude of attending physicians. At Kuma Hospital, a brochure that explains the safety and favorable outcomes of active surveillance is given to patients who have nodules suspected of being PTMC on ultrasound before the cytological examination rather than after cytology. A second brochure 
is given to patients who undergo active surveillance for a long period, to eliminate or alleviate their anxiety. The Memorial Sloan Kettering Cancer Center and the Korean team established an aid named "Thyroid Cancer Treatment Choice" to support conversations between physicians and patients (41). According to their report, patients who were given the aid were more likely to choose active surveillance than those without the aid. A recent study from the Asan Medical Center in Korea also reported that the quality of life of patients who received active surveillance was better than that of patients who underwent thyroid lobectomy (42).

\section{Education to the patients in order to prevent dropout}

Dropout is an important point for physicians. They are afraid of the dropout of patients from active surveillance, because a portion of PTMC should grow and turn to be clinical. In order to avoid dropout, education to the patients is very important (43). We routinely perform cytological examination for suspicious nodules and tell them the truth, which can prevent dropout of patients at least in part. Further, we send a letter to the patients who do not visit to our hospital for longer than 18 months asking them to have a checkup.

\section{Biological markers to predict PTMC growth}

Hirokawa et al. (44) showed that the Ki-67 labeling index in enlarged PTMCs was significantly higher than that in PTMCs that did not enlarge. However, since the expression of $\mathrm{Ki}-67$ is very heterogeneous, this strategy cannot be used on cytological specimens. At present, no molecular or pathological markers are available to predict PTMC progression using cytological specimens. The combination of $B R A F$ and TERT mutation is known as a predictor of poor prognosis for clinical PTCs. However, the incidences of $B R A F$ mutation were similar in PTMCs regardless of disease progression, and none of the PTMCs, including those that showed enlargement during active surveillance, had TERT mutations (45). To date, continuous active surveillance is the only method to identify PTMCs with constant growth.

\section{Conclusions}

Active surveillance is an excellent strategy for patients with low-risk PTMCs; it has exhibited oncological safety, low incidences of unfavorable events, and lower medical costs compared to immediate surgery. The active surveillance strategy is suitable as the first-line management for PTMCs (46-48). In Japan, based on a questionnaire survey to member institutions of the JAES or Japanese Society of Thyroid Surgery, 53.8\% of patients underwent active surveillance during successive three months (49), indicating that this strategy is filtering into Japanese institutions. Authors hope that active surveillance will be further accepted as a good management strategy of low-risk PTMC in the world.

\section{Acknowledgments}

Funding: None.

\section{Footnote}

Provenance and Peer Review: This article was commissioned by the Guest Editor (Kennichi Kakudo) for the series "Asian and Western Practice in Thyroid Pathology: Similarities and Differences" published in Gland Surgery. The article was sent for external peer review organized by the Guest Editor and the editorial office.

Conflicts of Interest: Both authors have completed the ICMJE uniform disclosure form (available at http://dx.doi. org/10.21037/gs-2019-catp-03). The series "Asian and Western Practice in Thyroid Pathology: Similarities and Differences" was commissioned by the editorial office without any funding or sponsorship. The authors have no other conflicts of interest to declare.

Ethical Statement: The authors are accountable for all aspects of the work in ensuring that questions related to the accuracy or integrity of any part of the work are appropriately investigated and resolved.

Open Access Statement: This is an Open Access article distributed in accordance with the Creative Commons Attribution-NonCommercial-NoDerivs 4.0 International License (CC BY-NC-ND 4.0), which permits the noncommercial replication and distribution of the article with the strict proviso that no changes or edits are made and the original work is properly cited (including links to both the formal publication through the relevant DOI and the license). 
See: https://creativecommons.org/licenses/by-nc-nd/4.0/.

\section{References}

1. Ito Y, Miyauchi A. Prognostic factors and therapeutic strategies for differentiated carcinomas of the thyroid. Endocr J 2009;56:177-92.

2. Takebe K, Date M, Yamamoto N. Mass screening for thyroid cancer with ultrasonography. Karkinos 1994;7:309-17.

3. Miyauchi A, Ito $\mathrm{Y}$, Oda H. Insights into the management of papillary microcarcinoma of the thyroid. Thyroid 2018;28:23-31.

4. Miyauchi A. Clinical trials of active surveillance of papillary microcarcinoma of the thyroid. World J Surg 2016;40:516-22.

5. Takami H, Ito Y, Okamoto T, et al. Therapeutic strategy for differentiated thyroid carcinoma in Japan based on a newly established guideline managed by Japanese Society of Thyroid Surgeons and Japanese Association of Endocrine Surgeons. World J Surg 2011;35:111-21.

6. Japanese Society of Thyroid Surgery, Japan Association of Endocrine Surgeons. Guidelines for the Management of Thyroid Tumors 2nd ed. Tokyo, Japan, 2018.

7. Haugen BR, Alexander EK, Bible KC, et al. 2015 American Thyroid Association management guidelines for adult patients with thyroid nodules and differentiated thyroid cancer: The American Thyroid Association guidelines task force on thyroid nodules and differentiated thyroid cancer. Thyroid 2016;26:1-133.

8. Davies L, Welch HG. Increasing incidence of thyroid cancer in the United States, 1973-2002. JAMA 2006;295:2164-7.

9. Davies L, Welch HG. Current thyroid cancer trends in the United States. JAMA Otolaryngol Head Neck Surg 2014;140:317-22.

10. Ahn HS, Kim HJ, Welch HG. Korea's thyroid-cancer "epidemic" — Screening and overdiagnosis. N Engl J Med 2014;371:1765-7.

11. Vaccarella S, Franceschi W, Bray F, et al. Worldwide thyroid-cancer epidemic? The increasing impact of overdiagnosis. N Engl J Med 2016;375:614-7.

12. Oda H, Miyauchi A, Ito Y, et al. Incidences of unfavorable events in the management of low-risk papillary microcarcinoma of the thyroid by active surveillance versus immediate surgery. Thyroid 2016;26:150-5.

13. Ito $\mathrm{Y}$, Miyauchi A, Oda H, et al. Revisiting low-risk thyroid papillary microcarcinomas resected without observation: Was immediate surgery necessary? World J Surg 2016;40:523-8.

14. Ito $Y$, Uruno T, Nakano K, et al. An observation trial without surgical treatment in patients with papillary microcarcinoma of the thyroid. Thyroid 2003;13:381-7.

15. Ito $\mathrm{Y}$, Miyauchi A, Inoue $\mathrm{H}$, et al. An observational trial for papillary thyroid microcarcinoma in Japanese patients. World J Surg 2010;34:28-35.

16. Ito Y, Miyauchi A, Kihara M, et al. Patient age is significantly related to the progression of papillary microcarcinoma of the thyroid under observation. Thyroid 2014:24:27-34.

17. Sugitani I, Toda K, Yamada K, et al. Three distinctly different kinds of papillary thyroid microcarcinoma should be recognized: Our treatment strategies and outcomes. World J Surg 2010;34:1222-31.

18. Sakai T, Sugitani I, Ebina A, et al. Active surveillance for T1bN0M0 papillary thyroid carcinoma. Thyroid 2019;29:59-63.

19. Brito JP, Ito Y, Miyauchi A, et al. A clinical framework to facilitate risk stratification when considering an active surveillance alternative to immediate biopsy and surgery in papillary microcarcinoma. Thyroid 2016;26:144-9.

20. Tuttle RM, Fagin JA, Minkowitz G, et al. Natural history and tumor volume kinetics of papillary thyroid cancers during active surveillance. JAMA Otolaryngol Head Neck Surg 2017;143:1015-20.

21. Kwon H, Oh HS, Kim M, et al. Active surveillance for patients with papillary thyroid microcarcinoma: A single center's experience in Korea. J Clin Endocrinol Metab 2017; 102:1917-25.

22. Oh HS, Ha J, Kim HI, et al. Active surveillance of low-risk papillary thyroid microcarcinoma: A multi-center cohort study in Korea. Thyroid 2018;28:1587-94.

23. Sanabria A. Active surveillance in thyroid microcarcinoma in a Latin-American cohort. JAMA Otolaryngol Head Neck Surg 2018;144:947-8.

24. Smulever A, Pitoia F. Active surveillance in papillary thyroid carcinoma: Not easily accepted but possible in Latin America. Arch Endocrinol Metab 2019;63:462-9.

25. Molinaro E, Campopiano MC, Peruzzi L, et al. Active surveillance in papillary thyroid microcarcinoma is feasible and safe: Experience at one Italian single center. J Clin Endocrinol Metab 2020. doi:10.1210/clinem/dgz113.

26. Miyauchi A, Kudo T, Ito Y, et al. Estimation of the lifetime probability of disease progression of papillary microcarcinoma of the thyroid during active surveillance. Surgery 2018;163:48-52. 
27. Miyauchi A, Kudo T, Miya A, et al. Prognostic impact of serum thyroglobulin doubling-time under thyrotropin suppression in patients with papillary thyroid carcinoma who underwent total thyroidectomy. Thyroid 2011;21:707-16.

28. Miyauchi A, Kudo T, Ito Y, et al. Natural history of papillary thyroid microcarcinoma: Kinetic analyses on tumor volume during active surveillance and before presentation. Surgery 2019;165:25-30.

29. Ito Y, Miyauchi A, Kudo T, et al. Kinetic analysis of growth activity in enlarging papillary thyroid microcarcinomas. Thyroid 2019;29:1765-73.

30. Ito Y, Miyauchi A, Kudo T, et al. Effects of pregnancy on papillary microcarcinomas of the thyroid re-evaluated in the entire patient series at Kuma Hospital. Thyroid 2016;26:156-60.

31. Fukuoka O, Sugitani I, Ebina A, et al. Natural history of asymptomatic papillary thyroid microcarcinoma: Timedependent changes in calcification and vascularity during active surveillance. World J Surg 2016;40:529-37.

32. Oda H, Miyauchi A, Ito Y, et al. Comparison of the costs of active surveillance and immediate surgery in the management of low-risk papillary microcarcinoma of the thyroid. Endocr J 2017;64:59-64.

33. Lang BH, Wong CK. A cost-effectiveness comparison between early surgery and non-surgical approach for incidental papillary thyroid microcarcinoma. Eur J Endocrinol 2015;173:367-75.

34. Venkatesh S, Pasternak JD, Beninato T, et al. Cost-effectiveness of active surveillance versus hemithyroidectomy for micropapillary thyroid cancer. Surgery 2017;161:116-26.

35. Sugitani I, Fujimoto Y, Yamada K. Association between serum thyrotropin concentration and growth of asymptomatic papillary thyroid microcarcinoma. World J Surg 2014;38:673-8.

36. Kim HI, Jang HW, Ahn HS, et al. High serum TSH level is associated with progression of papillary thyroid microcarcinoma during active surveillance. J Clin Endocrinol Metab 2018;103:446-51.

37. Nickel B, Brito JP, Barratt A, et al. Clinicians' views on management and terminology for papillary thyroid microcarcinoma: A qualitative study. Thyroid 2017;27:661-71.

38. Nickel B, Brito JP, Moynihan R, et al. Patients' experiences of diagnosis and management of papillary thyroid microcarcinoma: A qualitative study. BMC Cancer 2018;18:242.
39. Ito Y, Miyauchi A, Kudo T, et al. Trends in the implementation of active surveillance for low-risk papillary thyroid microcarcinomas at Kuma Hospital: Gradual increase and heterogeneity in the acceptance of this new management option. Thyroid 2018;28:488-95.

40. Davies L, Roman BR, Fukushima M, et al. Patient experience of thyroid cancer active surveillance in Japan. JAMA Otolaryngol Head Neck Surg 2019;145:363-70.

41. Brito JP, Moon JH, Zeuren R, et al. Thyroid cancer treatment choice: A pilot study of a tool to facilitate conversations with patients with papillary microcarcinomas considering treatment options. Thyroid 2018;28:1325-31.

42. Jeon MJ, Lee YM, Sung TY, et al. Quality of life in patients with papillary thyroid microcarcinoma managed by active surveillance or lobectomy: A cross-sectional study. Thyroid 2019;29:956-62.

43. Haser GC, Tuttle RM, Su HK, et al. Active surveillance for papillary thyroid microcarcinoma: New challenges and opportunities for the health care system. Endocr Pract 2016;22:602-11.

44. Hirokawa M, Kudo T, Ota H, et al. Pathological characteristics of low-risk papillary thyroid microcarcinoma with progression during active surveillance. Endocr J 2016;63:805-10.

45. Yabuta T, Matsuse M, Hirokawa M, et al. TERT promoter mutations were not found in papillary thyroid microcarcinomas that showed disease progression on active surveillance. Thyroid 2017;27:1206-7.

46. Miyauchi A, Ito Y. Conservative surveillance management of low-risk papillary thyroid microcarcinoma. Endocrinol Metab Clin North Am 2019;48:215-26.

47. Ito Y, Miyauchi A. Active surveillance as first-line management of papillary microcarcinoma. Ann Rev Med 2019;70:369-79.

48. Ito Y, Miyauchi A, Oda H. Low-risk papillary microcarcinoma of the thyroid: A review of active surveillance trials. Eur J Surg Oncol 2018;44:307-15.

49. Sugitani I, Ito Y, Miyauchi A, et al. Active surveillance versus immediate surgery: questionnaire survey on the current treatment strategy for adult patients with lowrisk papillary thyroid microcarcinoma in Japan. Thyroid 2019;29:1563-71.

Cite this article as: Ito Y, Miyauchi A. Active surveillance of low-risk papillary thyroid microcarcinomas. Gland Surg 2020;9(5):1663-1673. doi: 10.21037/gs-2019-catp-03 